\title{
Thermal Model of the Casting Process of Functionally Graded Composites
}

\author{
R. Przyłucki · S. Golak
}

Received: 15 April 2012 / Accepted: 17 January 2013 / Published online: 3 February 2013

C The Author(s) 2013. This article is published with open access at Springerlink.com

\begin{abstract}
In this article, a sophisticated thermal model of the casting process of functionally graded composites is presented. Electromagnetic buoyancy is used to separate the reinforcement particles. The model takes into account the nonuniform distribution of heat sources characteristic for the process, and also the basic mechanisms of heat transport: conduction and convection. The model allowed the authors to estimate the influence of heat phenomena on the trajectories of the reinforcement particles migrating in the matrix during the process. The methodology is illustrated by an example.
\end{abstract}

Keywords Electromagnetic buoyancy · Functionally graded composites · Natural convection

\section{Introduction}

In recent years, scientific research centers dealing with composite materials have substantially developed the research on properties and manufacturing technologies of a subgroup of these materials, namely, functionally graded composites or FGCs. Ensuring a desired concentration gradient, granulation, or type of reinforcement in the composite matrix gives completely new possibilities of material design. The attractiveness of this type of composite lies in the possibility of a gradual spatial change in their properties such as thermal conductivity, electrical conductivity, and hardness. The main advantage of such a change is avoidance of risks connected with stress formation at the boundaries of materials with substantially different properties. Due to their

R. Przyłucki (西) · S.Golak

Silesian University of Technology, Krasińskiego 8, 40-019 Katowice, Poland

e-mail: roman.przylucki@polsl.pl 
characteristics, functionally graded composites are of great interest to the automotive, aerospace, and medical industries.

Many production methods of these materials have already been developed. In the case of popular metal matrix composites, the most effective ones are casting techniques in which various physical phenomena are used to move the reinforcement particles in the desired direction. The most frequently used method in this group is centrifugal casting which utilizes the difference between the densities of the particles and the matrix [1,2]. The authors of this paper are working on a project in which electromagnetic buoyancy is used to separate the reinforcement particles [3]. This phenomenon exploits the influence of electromagnetic forces on the conductive metal matrix, which results in the migration of the non-conductive reinforcement particles in the direction opposite to these forces [4,6-8]. This technique gives more freedom as to the choice of the material composition of the reinforcement and the matrix. It also yields the spatial distributions in the reinforcement that are impossible to achieve in other casting methods.

The basic problem encountered in this process is to obtain electromagnetic buoyancy and simultaneously to avoid a flow of the liquid metal which destroys the desired composite structure. This undesirable metal flow is caused mainly by a non-uniform electromagnetic force density distribution. The authors managed to minimize this by adding special conductive elements to the mold and using specific shapes of the inductor. However, there is one more mechanism, independent of the force field, which makes the metal move. It is thermal convection caused by a non-uniform temperature distribution in the cast, which in turn results from cooling of the cast after pouring it into the mold and also from the presence of volumetric heat sources which are the effect of the electromagnetic field.

A strong electromagnetic field makes it impossible to carry out standard temperature measurements with a thermocouple, while the inductor does not allow a pyrometric measurement of the mold surface. For this reason the only way to identify the phenomena taking place in this process and to assess the influence of convection on the disturbance of the process of particles separation is creating a model of the process and conducting computer simulations.

\section{Real Model}

The research was conducted for a process of casting into a plaster mold of an aluminum bush reinforced with $\mathrm{SiC}$ particles at the outer wall. The shape of the cast corresponds to the popular applications of composite materials such as pistons in compressors and combustion engines. The dimensions of the bush, that is, the diameter of $65 \mathrm{~mm}$, height of $65 \mathrm{~mm}$, and wall thickness of $8 \mathrm{~mm}$, are typical dimensions of potential products.

The application of the standard mold and inductor would have resulted in nonuniformity of the magnetic field (especially at the ends of the cast), and consequently in non-uniformity of the electromagnetic force field (Fig. 1).

In order to obtain a uniform electromagnetic field at the ends of the cast, the mold was provided with conductive elements of the same conductivity as the liquid cast. In addition, the effect of a higher field strength in the middle of the inductor was 

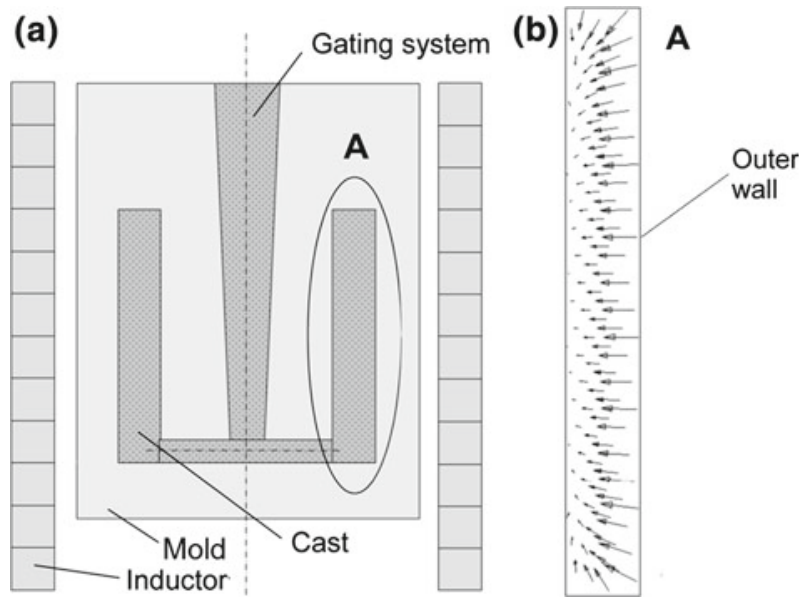

Fig. 1 (a) Standard casting system and (b) electromagnetic field distribution

eliminated by the application of a gradient inductor with intercoil spacing increasing toward the middle of the inductor. A current of $500 \mathrm{~A}$ and $1000 \mathrm{~Hz}$ frequency ensured a penetration depth equal to the thickness of the bush wall.

\section{Calculation Model}

The bush casting setup was a two-dimensional (2D) geometric model. As can be seen in Fig. 2, adopting this type of geometry meant that the channels joining the cast with the vertical inlet channel were not taken into account, which was possible because they do not influence thermal processes significantly. Still, these channels ensure better filling of the mold during the solidification of the cast. The shrinkage of the material in the mold is smaller as a result of continuous flow of the metal from the inlet channel.

\section{Electromagnetic Field Analysis}

The analysis of the electromagnetic field was carried out based on Maxwell's equations supplemented by the generalized Ohm's law. The calculations were simplified by the transition from time-domain analysis to the symbolic analysis.

$$
\nabla \times\left(\frac{1}{\mu} \nabla \times \boldsymbol{A}\right)+\mathrm{j} \omega \sigma \boldsymbol{A}=\boldsymbol{J}_{\mathrm{s}}
$$

where $\mu, \sigma$ are the magnetic permeability and the conductivity of the matrix, respectively. $\boldsymbol{A}$ is the magnetic vector potential, $\omega$ is the angular frequency, and $\boldsymbol{J}_{\mathrm{S}}$ is the source current density.

Electromagnetic field analysis was based on the expression using the magnetic vector potential $\boldsymbol{A}$ (Eq. 1) which is commonly used for steady-state electromagnetic problems $[9,10]$. The professional program Flux $2 \mathrm{~d}$ based on the finite element method 
Fig. 2 Calculation model of the casting setup (dimensions in $\mathrm{mm})$

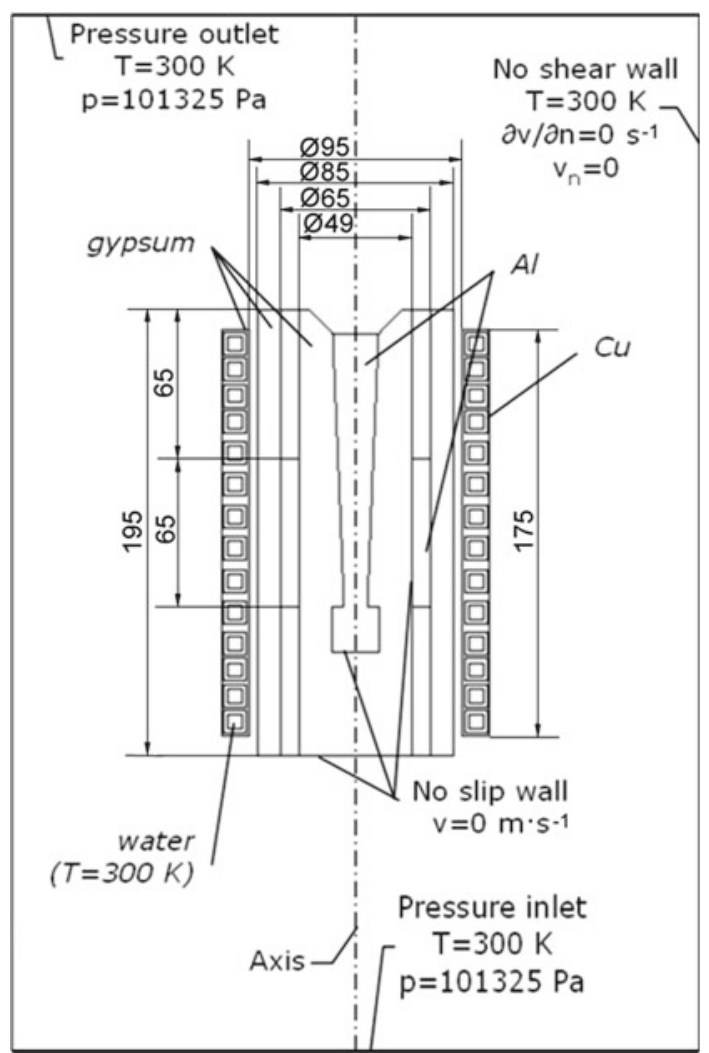

(FEM) with authors' procedures was used for the electromagnetic field calculation. The triangular mesh for electromagnetic field analysis had 211559 nodes.

$$
\begin{aligned}
\boldsymbol{B} & =\nabla \times \boldsymbol{A} \\
\boldsymbol{J} & =-\mathrm{j} \omega \sigma \boldsymbol{A}
\end{aligned}
$$

where $\boldsymbol{B}$ is the magnetic induction and $\boldsymbol{J}$ is the current density.

In order to allow a one-way coupling (weak coupling) of the electromagnetic model with the hydrodynamic model [3], two elements were not taken into account, i.e., the effect of temperature on the conductivity of the matrix (because of the small range of its changes during the action of the electromagnetic field) and the density of the current caused by metal flow (because the magnetic Reynolds number for the problem is smaller then $10^{-2}$ ). The electromagnetic induction $\boldsymbol{B}$ and the eddy current density $\boldsymbol{J}$ were determined from Eq. 1 after taking into account the dependences described by Eqs. 2 and 3.

$$
\boldsymbol{f}_{\mathrm{e}}=\frac{1}{2} \operatorname{Re}\left(\boldsymbol{J} \times \boldsymbol{B}^{*}\right)
$$


where $f_{\mathrm{e}}$ is the volumetric density of electromagnetic force and $\boldsymbol{B}^{*}$ is the complex conjugate of $\boldsymbol{B}$.

Equations 1, 2, and 3 were solved by the finite element method in two-dimensional axisymmetric space. The volumetric density of the time-average electromagnetic force acting on the molten metal was determined from Eq. 4.

$$
s=\frac{J^{2}}{\sigma}
$$

where $s$ is the volumetric heat density.

The electromagnetic field penetrating the charge causes eddy currents which are the source of heat. The distribution of heat sources in the charge is strongly dependent on the intensity and frequency of the inductor current. Equation 5 allows the determination of volumetric heat sources induced in the charge.

\section{Thermal Field Analysis}

The hydrodynamic model of the whole process, which also took into account the flows of air around the mold, allowed a direct calculation of the heat exchange between the mold and ambient air, which would not be possible in the case of the simplification where the heat exchange coefficient is used at the outside edges of the mold.

$$
\frac{\partial}{\partial t}\left(\rho_{\mathrm{m}} h\right)+\nabla\left(\mathbf{v} \rho_{\mathrm{m}} h\right)=\nabla(k \nabla T)+s
$$

where

$$
h=\int_{T_{\text {ref }}}^{T} c_{p} \mathrm{~d} T
$$

where $\rho_{\mathrm{m}}$ is the density of the matrix, $h$ is the enthalpy, $v$ is the fluid velocity, $k$ is the thermal conductivity, $T$ is the temperature, $c_{p}$ is the specific heat, and $T_{\text {ref }}$ is the initial temperature of the set (in the analyzed case, $298 \mathrm{~K}$ ).

Heat transport was calculated from the energy equation (Eq. 6).

\section{Flow Field Analysis}

The fluid flow in the closed system is the result of a non-uniform distribution of the acting forces $f_{\mathrm{e}}[3]$,

$$
\nabla \times f_{\mathrm{e}}=0
$$

Since the gravitational field is uniform in technological scale, if the liquid is to remain at rest, the electromagnetic force field has to be irrotational. The irrotation condition 
of the force field is described by Eq. 8. Since it is virtually impossible to obtain a perfectly uniform distribution of electromagnetic forces in a finite-length casting, an initiation of liquid metal flow is unavoidable;

$$
\begin{aligned}
\rho_{f}\left(\frac{\partial \boldsymbol{v}}{\partial t}+\boldsymbol{v} \nabla \boldsymbol{v}\right) & =-\nabla p+\eta \nabla^{2} \boldsymbol{v}+\mathrm{f}_{\mathrm{e}}-\rho_{m 0} \beta\left(T-T_{0}\right) \boldsymbol{g} \\
\nabla \boldsymbol{v} & =0
\end{aligned}
$$

where $\boldsymbol{v}$ is the matrix velocity, $\eta$ is the dynamic viscosity of the matrix, $\rho_{\mathrm{m} 0}$ is the density of the matrix for the reference temperature, $\beta$ is the thermal expansion coefficient, $T_{0}$ is the reference temperature, $p$ is the pressure, and $g$ is the gravitational acceleration.

The flow is described by the Navier-Stokes and continuity equations for incompressible fluid described by Eqs. 9 and 10. The program Ansys Fluent based on FVM was used for the hydrodynamic calculations. The triangular mesh for the flow and temperature field analysis had 168163 cells. The threshold for an absolute, scaled convergence criterion for continuity and a velocity was set at a value of $10^{-5}$. The time step size of the simulation equaled $10^{-3} \mathrm{~s}$.

$$
\boldsymbol{f}_{\mathrm{k}}=\rho_{\mathrm{m} 0} \beta\left(T-T_{0}\right) \boldsymbol{g}
$$

where $f_{\mathrm{k}}$ is the volumetric density of the convection force.

As the channels carrying the liquid metal from the inlet channel to the cast are not taken into account in 2D modeling, the bush becomes a closed system. In such a case, when the density-temperature dependence (which is a basic mechanism of convective motion) is introduced to the model, unnaturally large empty spaces will occur in the cast during the solidification stage. In order to achieve the proper filling of the mold after solidification and at the same time to model the convective motion, the authors decided to use the Boussinesq approximation. This dependence was introduced as the last part of the momentum equation (Eq. 9). The Boussinesq approximation in the form presented by Eq. 11 can be applied because the condition $\beta\left|T-T_{0}\right|<<1$ is fulfilled.

\section{Particle Motion}

A reinforcement particle immersed in the liquid metal in the electromagnetic field is affected by the resultant of the Stokes drag force $\boldsymbol{F}_{\mathrm{d}}$, the gravitational force $\boldsymbol{F}_{\mathrm{g}}$, and the electromagnetic force $\boldsymbol{F}_{\mathrm{e}}$.

$$
\begin{aligned}
& \boldsymbol{F}_{\mathrm{p}}=\boldsymbol{F}_{\mathrm{d}}+\boldsymbol{F}_{\mathrm{g}}+\boldsymbol{F}_{\mathrm{e}} \\
& \boldsymbol{F}_{\mathrm{d}}=3 \pi \eta d\left(\boldsymbol{v}-\boldsymbol{v}_{\mathrm{p}}\right)
\end{aligned}
$$

where $\boldsymbol{F}_{\mathrm{p}}$ is the net force acting on a particle, $\boldsymbol{F}_{\mathrm{d}}$ is the Stokes force, $\boldsymbol{F}_{\mathrm{g}}$ is the gravitational force, $\boldsymbol{F}_{\mathrm{e}}$ is the electromagnetic force, and $\boldsymbol{v}_{\mathrm{p}}$ is the particle velocity. 
The Stokes drag force acting on a spherical particle of diameter $d$ in a fluid of dynamic viscosity $\eta$ for small Reynolds numbers is described by Eq. 13. The total balance of the forces is described by Eq. 12 .

$$
\boldsymbol{F}_{\mathrm{g}}=\pi d^{3} \boldsymbol{g}\left(\rho_{\mathrm{p}}-\rho_{\mathrm{m}}\right) / 6
$$

where $\rho_{\mathrm{p}}$ is the particle density and $\rho_{\mathrm{m}}$ is the matrix density.

The action of gravity presented by Eq. 14 is the result of the difference between the densities of the matrix $\rho_{\mathrm{m}}$ and the reinforcement particles $\rho_{\mathrm{p}}$.

$$
\boldsymbol{F}_{\boldsymbol{e}}=-\pi d^{3} \boldsymbol{f}_{\mathrm{e}} / 8
$$

In the metal matrix composites reinforced with ceramic particles, conductivity of which is negligibly small relative to the conductivity of the matrix, the electromagnetic force acting on a particle can be determined from the dependence (Eq. 15) [11].

$$
\frac{\mathrm{d} v_{\mathrm{p}}}{\mathrm{d} t}=\frac{1}{\rho_{\mathrm{p}}}\left(\frac{18 \eta\left(v-v_{\mathrm{p}}\right)}{d^{2}}+g\left(\rho_{\mathrm{p}}-\rho_{\mathrm{m}}\right)-\frac{3}{4} f_{\mathrm{e}}\right)
$$

The trajectory of a particle can be computed by integrating the force balance on the particle described by Eq. 16. For the integration the trapezoidal rule integration method was used.

$$
v_{\mathrm{p}}=-d^{2} \boldsymbol{f}_{\mathrm{e}} \eta^{-1} / 24
$$

By equating $\boldsymbol{F}_{\mathrm{e}}$ with $\boldsymbol{F}_{\mathrm{d}}$, the maximum velocity of the particle (in the desired direction) that can be obtained as a result of the action of the electromagnetic force can be derived as in Eq. 17.

\section{Determination of the Initial Heating Temperature of the Mold}

A popular technique used in foundry engineering which allows proper filling of the mold is its initial heating before the pouring starts. The choice of the optimal temperature of the mold for typical aluminum products has great influence on the structure and the properties of the cast. It is generally assumed that a lower temperature of the mold results in faster cooling of the cast, which means that a more desired, fine-grained structure is obtained.

With functionally graded composites the range of minimum initial temperatures of the mold is limited because after the pouring takes place, the metal must remain liquid for a period of time to allow the particles to be moved by the forces of electromagnetic buoyancy, in which way the desired spatial distribution of the reinforcement is obtained. The time can be determined on the basis of the electromagnetic force distribution and the dependence between the particle speed and the density of the forces (Eq. 17). For the bush considered, the time is about $5 \mathrm{~s}$ for the $25 \mu \mathrm{m}$ particles. 
Fig. 3 Volumetric heat sources (frequency of $1000 \mathrm{~Hz}$ )

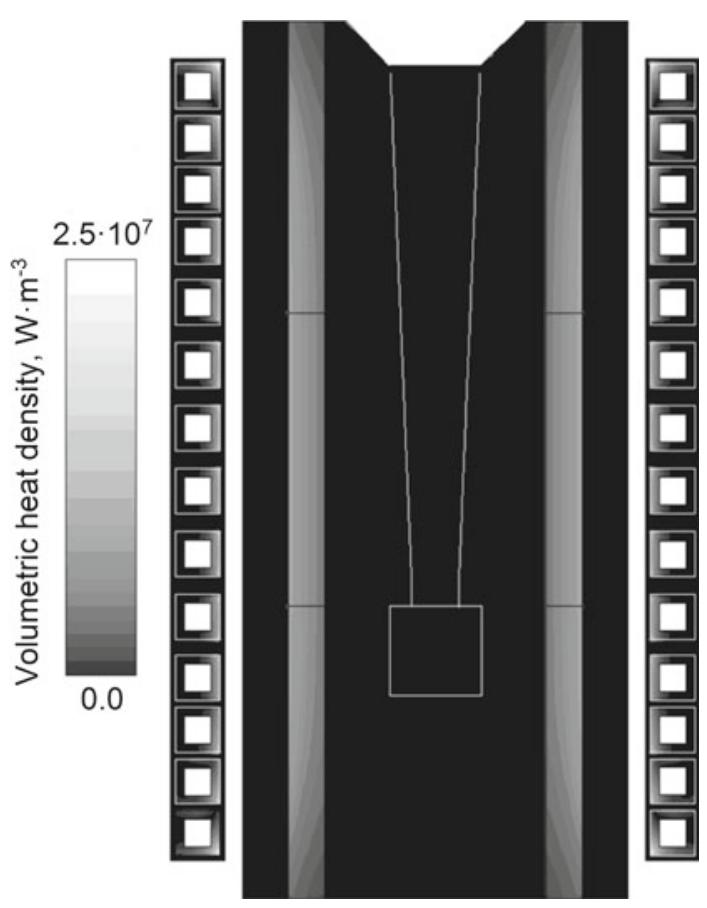

The simulations were carried out taking into account the volumetric sources of heat generated by eddy currents induced by the electromagnetic field (Fig. 3). The diagram shows that the frequency of the inductor current, optimized in the previous studies in terms of the distribution of the force field, causes a relatively uniform distribution of density of the heat generated in the cast.

A series of numerical simulations made it possible to determine the minimum mold temperature of $805 \mathrm{~K}$. It ensures that the temperature at any point of the cast will not drop below the solidification temperature during the first $5 \mathrm{~s}$ after the pouring (Fig. 4a). At the same time, this temperature ensures that after the particles are moved, the temperature of the whole matrix drops below the solidification temperature relatively quickly (Fig. 4b). The research was conducted for the typical pouring temperature of $1073 \mathrm{~K}$.

\section{Determination of the Particle Trajectories}

As mentioned above, the basic goal of this study was to determine whether the convective flow of the liquid metal has any influence on the trajectory of the migrating particles, and at the same time, on the final spatial distribution of the reinforcement. The research was conducted for the initial heating temperature of the mold which was determined in the previous section $(805 \mathrm{~K})$.

The research was done for three cases: 

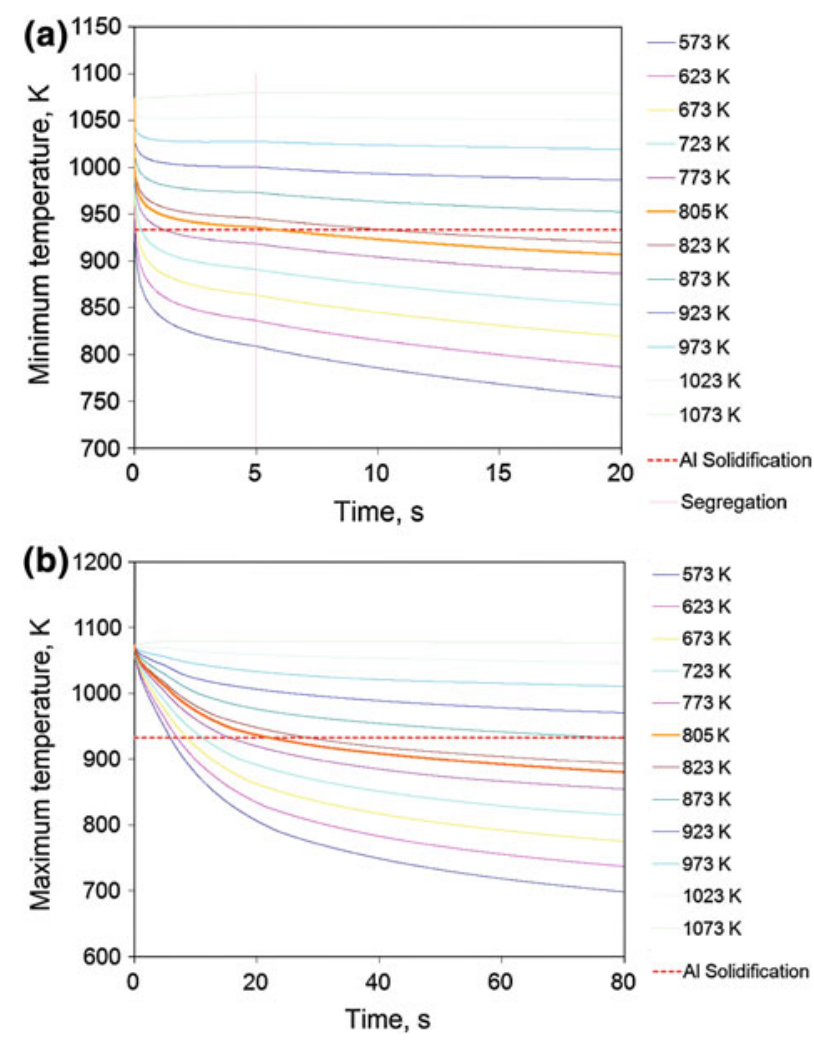

Fig. 4 Dependence of (a) minimum and (b) maximum temperature of the cast with time for various initial heating temperatures of the mold

- The real casting setup, where, despite the inductor optimization, some nonuniformity of the electromagnetic field is manifested which results in the movement of the liquid metal. The other cause of this undesirable movement is convective motion.

- The ideal casting setup in terms of the electromagnetic field uniformity. The only cause of the metal flow is convection.

- The ideal casting setup in terms of the electromagnetic field uniformity without modeling of the convective motion. The only forces acting on the particles are gravity and electromagnetic buoyancy. No convection appears.

The particles, $50 \mu \mathrm{m}$ in diameter, are moved from the inner wall to the outer wall of the bush within $1.1 \mathrm{~s}$, the time being so short that even in the real setup with non-uniform electromagnetic force field the movement of the liquid metal is insignificant and does not disturb the particle trajectories. For this reason the trajectories for the particular cases are quite similar (Fig. 5).

As can be seen from Eq. 17, the particles of $25 \mu \mathrm{m}$ in diameter gain a four times lower speed at the same density of electromagnetic forces, which significantly increases the migration time of the particles which are the most distant from the 
Fig. 5 Trajectories of $50 \mu \mathrm{m}$ particles (arrows - direction of movement of particles): (a) real setup (non-uniform $f_{m}$ force field, convection), (b) uniform $f_{m}$ force field, convection, and (c) uniform $f_{m}$ force field, no convection

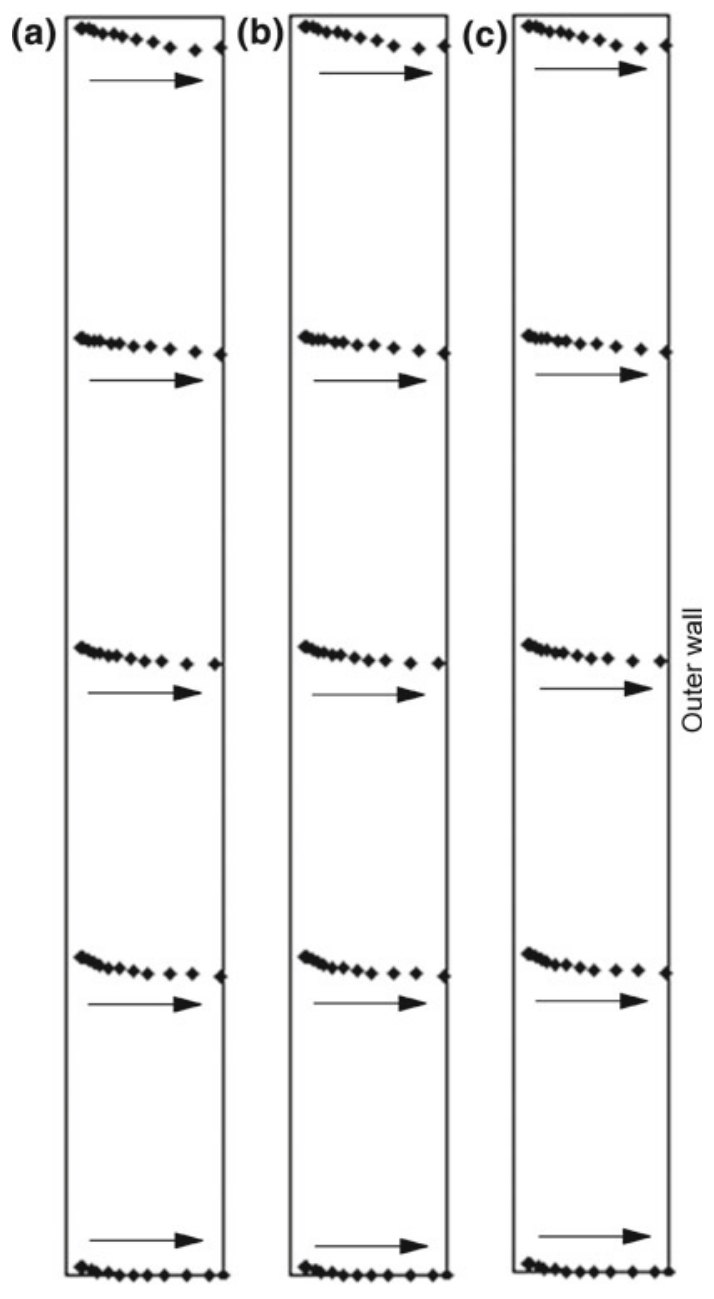

destination area (up to $5 \mathrm{~s}$ ), in this way allowing time for the liquid metal to gain speed. In the case of the real setup, it can be seen (Fig. 6a) that a non-uniform force distribution causes the movement of the liquid metal to be sufficiently intense to significantly disturb the trajectory of the particles in the upper area of the cast.

The comparison of the trajectories, with (Fig. 6b) and without (Fig. 6c) convection taken into account, shows that only a slight difference can be noticed in the marked area.

The influence of convection becomes more visible for the $10 \mu \mathrm{m}$ particles which move six times slower than the $25 \mu \mathrm{m}$ particles (Fig. 7). The vertical motion of the metal (Fig. 7d), resulting from the liquid metal being cooled by the mold, at first pushes the particles down, then pushes them up, and at the end, it pushes them down. 
Fig. 6 Trajectories of $25 \mu \mathrm{m}$ particles (black arrows-direction of movement of particles, white arrows - change of trajectory): (a) real setup (non-uniform $f_{m}$ force field, convection), (b) uniform $f_{m}$ force field, convection, and (c) uniform $\boldsymbol{f}_{m}$ force field, no convection (a)

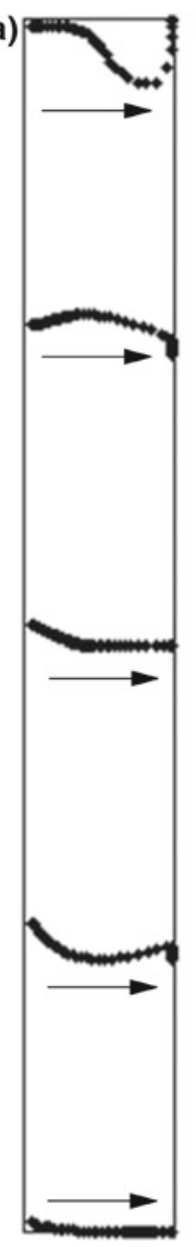

(b)

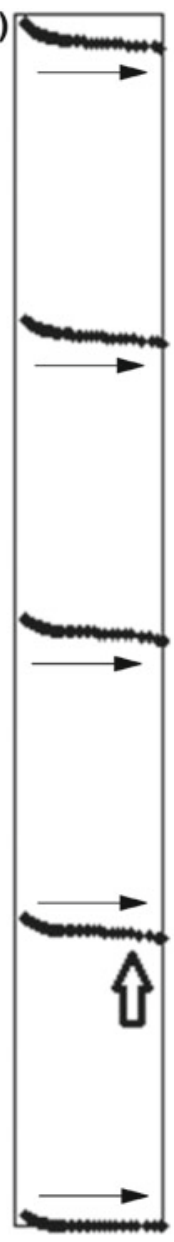

(c)

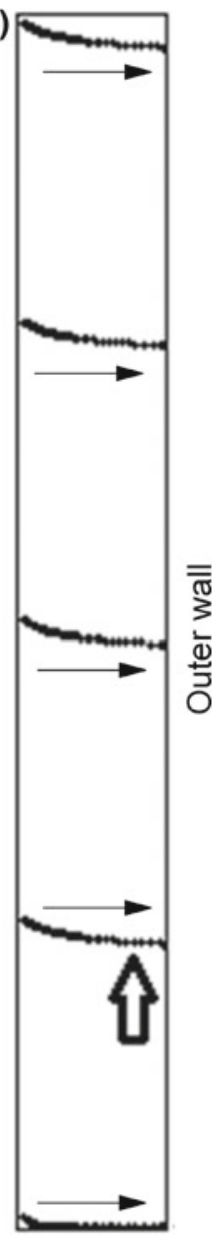

\section{Conclusions}

The strong electromagnetic fields make it difficult to take temperature measurements during the casting of functionally graded composites. For this reason in order to understand heat phenomena occurring during this kind of casting, a mathematical model of such a process was created. The authors' efforts made so far to ensure the greatest possible uniformity of the electromagnetic force field allowed them to minimize the influence of this factor. This paper shows that the liquid metal stability obtained through the specific structure of the mold and specific shapes of the inductor will not be disturbed by convective motion. The research showed that convection becomes a significant factor only for very small particles. Even then, however, it does not disturb the process of particle separation to the extent that would hinder obtaining the desired distribution of the reinforcement concentration. 


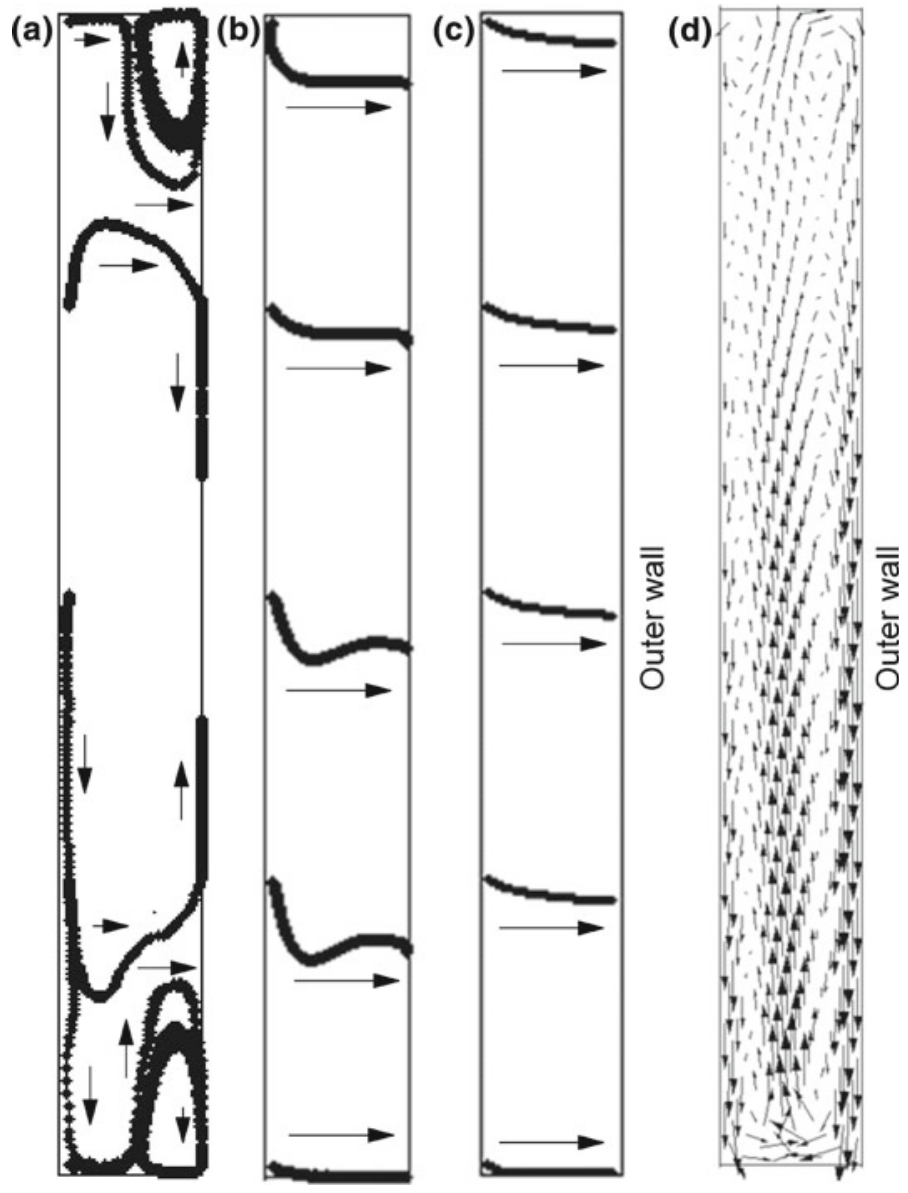

Fig. 7 Trajectories of $10 \mu \mathrm{m}$ particles (arrows - direction of movement of particles): (a) real setup (nonuniform $f_{m}$ force field, convection), (b) uniform $f_{m}$ force field, convection, (c) uniform $f_{m}$ force field, no convection, and (d) metal flow disturbing the particle movement for case (b)

On the basis of the characteristics of the vortices resulting from convection and the flow direction at the outer wall of the cast (Fig. 7d), it can be concluded that the main driving force cause of the metal movement (for a uniform force field) is its cooling by the mold and not a relatively small non-uniformity of the sources of the heat produced by the electromagnetic field (Fig. 3).

Acknowledgments This work was supported by the National Science Centre of Poland under Grant N N508 620940.

Open Access This article is distributed under the terms of the Creative Commons Attribution License which permits any use, distribution, and reproduction in any medium, provided the original author(s) and the source are credited. 


\section{References}

1. R. Zagorski, J. Sleziona, Arch. Mater. Sci. Eng. 28, 441 (2007)

2. A. Dolata-Grosz, J. Sleziona, J. Wieczorek, K. Pietrzak, Kompozyty 3, 125 (2003)

3. S. Golak, R. Przylucki, IEEE Trans. Magn. 47, 4701 (2011)

4. Z. Xu, T. Li, Y. Zhou, Metall. Mater. Trans. A 34, 1719 (2003)

5. C. Song, Z. Xu, J. Li, Appl. Sci. Compos. A 38, 427 (2006)

6. K. Takahashi, S. Taniguchi, J. Jpn. Inst. Light Met. 55, 483 (2005)

7. Y. Kanno, S. Taniguchi, S. Shimasaki, N. Yoshikava, J. Jpn. Inst. Light Met. 57, 12 (2007)

8. S. Golak, R. Zagorski, Metalurgija 52, 215 (2013)

9. R. Przyłucki, Przegląd Elektrotechniczny 84, 210 (2008)

10. M. Niklewicz, A. Smalcerz, Przegląd Elektrotechniczny 86, 333 (2010)

11. D. Leenov, A. Kolin, J. Chem. Phys. 22, 684 (1954) 